\title{
Adaptive Motion/Force Tracking Control for a Class of Nonholonomic Mechanical Systems
}

\author{
Wei Sun \\ School of Automation \\ Southeast University \\ Nanjing, Jiangsu 210096, China \\ Email: tellsunwei@sina.com
}

\author{
Yu-qiang $\mathrm{Wu}$ \\ Institute of automation \\ Qufu normal university \\ Qufu, Shandong 273165, China \\ Email:wyq@qfnu.edu.cn
}

\begin{abstract}
The motion/force tracking control of nonholonomic mechanical systems with affine constraints is investigated in this paper. By flexibly using the algebra processing technique, constraint forces are successfully canceled in the dynamic equations, and then an integral feedback compensation strategy and an adaptive scheme are applied to identify the dynamic uncertainty. The proposed controller ensures that the state of the closed-loop system asymptotically tracks the desired trajectory and the force tracking error has a controllable bound.
\end{abstract}

Key words-Tracking control; mechanical system; holonomic constraints; affine constraints.

\section{INTRODUCTION}

Nonholonomic constraints arise in many mechanical systems when there is a rolling or sliding contact, such as wheeled mobile robots, $n$-trailer systems, space robots, underwater vehicles, multi-fingered robotic hands, and so on. Although great progress ${ }^{[1-5]}$ has been made for nonholonomic systems during the last decades, controller design for these systems still has a challenge to control engineers.

It is worth pointing out that most existing results ${ }^{[6-9]}$ of nonholonomic systems aimed at the classic nonholonomic linear constraints. In fact, there is another large class of constraints which are affine in velocities, called affine constraints ${ }^{[10-11]}$, such as a boat on a running river with the varying stream, ball on rotating table with invariable angular velocity, underactuated mechanical arm, etc. In [10], T. Kai defined rheonomous affine constraints and explained a geometric representation method for them, and derived a necessary and sufficient condition for complete nonholonomicity of the rheonomous affine constraints. But the problems of controllability and stabilizability on the nonholonomic kinematic mechanical systems with affine constraints have not been systematically analysed up to now.

The tracking problem for mechanical systems, as a much more interesting issue in practice, is to make the entire state of the closed-loop system track to a given desired trajectory. It is also important to note that the literatures on the tracking problem of the nonholonomic systems with affine constraints are sparse at present. Hence, researching the tracking problem for such nonholonomic mechanical systems is an innovatory and significative work. In this paper, we establish the dynamical model of the nonholonomic control systems with affine constraints. Based on the asymptotic tracking idea for uncertain multi-input nonlinear systems, and the compensatory strategy, an adaptive tracking controller is designed such that the trajectory tracking error asymptotically tends to zero and the force tracking error is bounded with a controllable bound.

\section{System Description AND CONTROL Design}

\section{A. Dynamics Model}

According to Euler-Lagrangian formulation, equations of nonholonomic mechanical systems are described by

$$
M(q) \ddot{q}+V(q, \dot{q}) \dot{q}+G(q)=f+B(q) \tau,
$$

where $q=\left[q_{1}, \cdots, q_{n}\right]^{T}$ is the generalized coordinates, and $\dot{q}, \ddot{q} \in \mathrm{R}^{n}$ represent the generalized velocity vector, acceleration vector, respectively; $M(q) \in \mathrm{R}^{n \times n}$ is the inertia matrix; $V(q, \dot{q}) \dot{q} \in \mathrm{R}^{n}$ presents the vector of centripetal, Coriolis forces; $G(q) \in \mathrm{R}^{n}$ represents the vector of gravitational forces; $\tau$ denotes the $r$-vector of generalized control inputs; $B(q) \in \mathrm{R}^{n \times r}$ is a known input transformation matrix $(r<n)$ with full rank; $f \in \mathrm{R}^{n}$ denotes the vector of constraint forces.

Consider the situation where kinematic constraints are imposed, which represented by analytical relations between the generalized coordinates $q$ and velocity vector $\dot{q}$, it is can be described by

$$
J^{T}(q) \dot{q}=A(q),
$$

where $J(q)=\left[j_{1}(q), \cdots, j_{m}(q)\right] \in \mathrm{R}^{n \times m}$ is full of constraint matrix, $A(q)=\left[a_{1}(q), \cdots, a_{m}(q)\right]^{T} \in \mathrm{R}^{m}$ is known.

\section{B. Reduced Dynamics and State Transformation}

It's easy to find a fullrank matrix $S \in \mathrm{R}^{n \times(n-m)}$ satisfying

$$
J^{T}(q) S(q)=0 .
$$

Noticing that $S(q)$ is full of rank, there must exist a full-rank matrix $S_{1}(q) \in \mathrm{R}^{(n-m) \times n}$ satisfying $S_{1}(q) S(q)=I$, where $I$ is an identity matrix. If defining $\xi(t)=[q,-t]^{T}$, then (2) can be expressed concisely as

$$
\left[\begin{array}{ll}
J^{T}(q) & A(q)
\end{array}\right] \dot{\xi}=0 .
$$

For the sake of convenience, we define

$$
E(q)=\left[\begin{array}{cc}
S(q) & \eta(q) \\
0 & -1
\end{array}\right] \in \mathrm{R}^{(n+1) \times(n-m+1)},
$$


where $\eta(q) \in \mathrm{R}^{n}$ satisfies $J^{T}(q) \eta(q)=A(q)$. One can deduce that $E$ is a full of rank and satisfies

$$
\left[\begin{array}{ll}
J^{T}(q) & A(q)
\end{array}\right] E(q)=0 .
$$

From (4) and (5), we know that there exists an $(n-m+1)$ dimensional vector $\bar{z}$, such that

$$
\dot{\xi}=E \dot{\bar{z}}=\left[\begin{array}{cc}
S(q) & \eta(q) \\
0 & -1
\end{array}\right] \dot{\bar{z}},
$$

where $\dot{\bar{z}}=\left[\dot{z}^{T}, \dot{z}_{n-m+1}\right]^{T}, z=\left[z_{1}, \ldots, z_{n-m}\right]^{T}$.

In view of the relationship (6), one can obtain $\dot{z}_{n-m+1}=1$, and the generalized velocity vectors can be written as

$$
\dot{q}=S(q) \dot{z}+\eta(q) .
$$

$z$ corresponds to the internal state variable, and $(q, z)$ is sufficient to describe the constrained motion.

Substituting (7) into (1), the system (1) and (2) can be described clearly as

$$
\bar{M}(q) \ddot{z}+\bar{V}(q, \dot{q}) \dot{z}+\bar{G}(q, \dot{q})=J(q) \lambda+B(q) \tau,
$$

where $\bar{M}(q)=M(q) S(q)$ and $\bar{V}(q, \dot{q})=M(q) \dot{S}(q)+$ $V(q, \dot{q}) S(q), \bar{G}(q, \dot{q})=M(q) \dot{\eta}(q)+V(q, \dot{q}) \eta(q)+G(q)$.

\section{Error System Development}

In practice, the complexity and unpredictability of the structure of uncertainties usually appear in the dynamics of the mechanical systems, we assume that $\bar{M}(q), V(q, \dot{q})$ and $\bar{G}(q, \dot{q})$ are expressed in the form

$$
\begin{aligned}
\bar{M}(q) & =M_{0}(q)+\nabla M(q), \\
\bar{V}(q, \dot{q}) & =V_{0}(q, \dot{q})+\nabla V(q, \dot{q}), \\
\bar{G}(q, \dot{q}) & =G_{0}(q, \dot{q})+\nabla G(q, \dot{q}),
\end{aligned}
$$

where $M_{0}, V_{0}, G_{0}$, as the nominal matrices, are assumed to be known exactly, and $\nabla M, \nabla V, \nabla G$ represent the uncertainties in system matrices. Dynamic model (8) can be rewritten as

$M_{0}(q) \ddot{z}+V_{0}(q, \dot{q}) \dot{z}+G_{0}(\cdot)+\Phi_{u}(\cdot)=J(q) \lambda+B(q) \tau$,

where $\Phi_{u}(\cdot)=\nabla M(q) \ddot{z}+\nabla V(q, \dot{q}) \dot{z}+\nabla G(q, \dot{q}) \in \mathrm{R}^{n-m}$.

Pre-multiplying $S^{T}(q)$ on both sides of (9), and noting $J^{T}(q) S(q)=0$, the following transformed system is received:

$M_{1}(q) \ddot{z}+V_{1}(q, \dot{q}) \dot{z}+G_{1}(q, \dot{q})+\bar{\Phi}_{1}(q, \dot{q}, \dot{z}, \ddot{z})=B_{1}(q) \tau$,

where $M_{1}(q)=S^{T}(q) M_{0}(q), V_{1}(q, \dot{q})=S^{T}(q) V_{0}(q, \dot{q})$, $B_{1}(q)=S^{T}(q) B(q), \quad G_{1}(q, \dot{q})=S^{T}(q) G_{0}(q, \dot{q})$, $\bar{\Phi}_{1}(q, \dot{q}, \dot{z}, \ddot{z})=S^{T}(q) \Phi_{u}(q, \dot{q}, \dot{z}, \ddot{z})$. According to Masahiro Oya' statement [8], there exists a coordinate transformation $q=\Psi(z)$ such that $\Phi_{1}(z, \dot{z}, \ddot{z})=\left.\bar{\Phi}_{1}(q, \dot{q}, \dot{z}, \ddot{z})\right|_{q=\Psi(z)}$. Let $\Phi_{1}$ replace $\bar{\Phi}_{1}$ in above equation, the following is obtained:

$$
M_{1}(q) \ddot{z}+V_{1}(q, \dot{q}) \dot{z}+G_{1}(q, \dot{q})+\Phi_{1}(z, \dot{z}, \ddot{z})=B_{1}(q) \tau \text {. }
$$

The control objective of this paper is specified as: A given desired trajectory $z_{d}(t)$ satisfying that $z_{d}^{(i)}(t), i=0, \cdots, 4$ exist and are bounded, a desired constraint force $f_{d}(t)$ or a desired multiplier $\lambda_{d}(t)$, determine a adaptive control law for system (1), such that: (i) All the states of the closed-loop system are globally bounded. (ii) The position and velocity tracking error $z(t)-z_{d}(t), \dot{z}(t)-\dot{z}_{d}(t)$ converge to zero as $t \rightarrow \infty$, respectively.

The subsequent development is based on the assumption that $\Phi_{1}$ is an $\mathcal{C}^{2}$ nonlinear vector function. In order to solve the previous problem, we make the following assumptions:

Assumption 1[12] The matrix $M_{1}$ is symmetric, positive definite and satisfies

$$
a\|x\|^{2} \leq x^{T} M_{1}(x) x \leq \bar{a}(\|x\|)\|x\|^{2},
$$

where $a$ is a known positive constant, $\bar{a}(x)$ is a known positive function.

Assumption 2 If $q(t) \in \mathcal{L}_{\infty}$, then $\partial M_{1}(q) / \partial q$ exists and is bounded. Moreover, if $q(t), \dot{q}(t), \ddot{q}(t) \in \mathcal{L}_{\infty}$, then $V_{1}(q, \dot{q})$ and $\partial V_{1}(q, \dot{q}) / \partial q$ exist and are all bounded.

Next, we develop the following error system which will be used in the subsequent controller design and stability analysis

$$
\begin{aligned}
& e_{1}=z_{d}-z, \\
& e_{\lambda}=\lambda-\lambda_{d},
\end{aligned}
$$

where $e_{1} \in \mathrm{R}^{n-m}, e_{\lambda} \in \mathrm{R}^{m}$. To achieve the desired control objective, the following filtered tracking errors, denoted by $e_{2}, \rho \in \mathrm{R}^{n-m}$, are defined as

$$
\left\{\begin{aligned}
e_{2} & =\dot{e}_{1}+\alpha_{1} e_{1}, \\
\rho & =\dot{e}_{2}+\alpha_{2} e_{2},
\end{aligned}\right.
$$

where $\alpha_{1}>0, \alpha_{2}>0$ are designed constants.

In view of (9), (11) and (13), pre-multiplying $M_{0}$ on both sides of the second formula of (13), the following expression can be arrived at:

$$
\begin{aligned}
M_{0} \rho= & M_{0} \ddot{z}_{d}+V_{0} \dot{z}_{d}+G_{0}+\Phi_{u}-B \tau-J(q) \lambda+\alpha_{1} M_{0} \dot{e}_{1} \\
& +\alpha_{2} M_{0} e_{2}-V_{0} \dot{e}_{1} .
\end{aligned}
$$

By the expression (14), a control torque input is designed as:

$$
B \tau=M_{0} \ddot{z}_{d}+V_{0} \dot{z}_{d}+G_{0}-J(q) \lambda_{c}+S_{1}^{T} \mu,
$$

where the force term $\lambda_{c}$ is defined as $\lambda_{c}=\lambda_{d}-k_{\lambda} e_{\lambda}, k_{\lambda}$ is a constant of force control feedback gain, and $\mu(t) \in \mathrm{R}^{n-m}$ denotes a subsequently designed control term. Substituting (15) into (14), we can further get

$$
\begin{aligned}
M_{0} \rho= & \Phi_{u}+J(q)\left(\lambda_{c}-\lambda\right)-S_{1}^{T} \mu+\alpha_{1} M_{0} \dot{e}_{1} \\
& +\alpha_{2} M_{0} e_{2}-V_{0} \dot{e}_{1} .
\end{aligned}
$$

After pre-multiplying $S^{T}(q)$, noting $S^{T}(q) J(q)=0$ and $S_{1}(q) S(q)=I$, the above equation becomes

$$
M_{1} \rho=\Phi_{1}-\mu+\alpha_{1} M_{1} \dot{e}_{1}+\alpha_{2} M_{1} e_{2}-V_{1} \dot{e}_{1} .
$$

To facilitate the design of $\mu(t)$, differentiating (17) yields:

$$
M_{1} \dot{\rho}=\dot{\Phi}_{1}-\dot{\mu}-\dot{M}_{1} \rho+\Upsilon,
$$

where $\Upsilon=\alpha_{1} \dot{M}_{1} e_{2}+\alpha_{1} M_{1} \rho-\alpha_{1} \alpha_{2} M_{1} e_{2}-\alpha_{1}^{2} \dot{M}_{1} e_{1}-$ $\alpha_{1}^{2} M_{1} e_{2}+\alpha_{1}^{3} M_{1} e_{1}+\alpha_{2} \dot{M}_{1} e_{2}+\alpha_{2} M_{1} \rho-\alpha_{2}^{2} M_{1} e_{2}-\dot{V}_{1} e_{2}-$ $V_{1} \rho+\alpha_{2} V_{1} e_{2}+\alpha_{1} \dot{V}_{1} e_{1}+\alpha_{1} V_{1} e_{2}-\alpha_{1}^{2} V_{1} e_{1}$.

Based on the method of compensation for uncertain dynamic $^{[13]}, \mu(t)$ is designed as follows:

$\mu(t)=\left(k_{s}+1\right) e_{2}(t)-\left(k_{s}+1\right) e_{2}(0)+\int_{0}^{t}\left(\left(k_{s}+1\right) \alpha_{2} e_{2}(s)\right.$ 


$$
\left.+\hat{\Theta}(s) \operatorname{sgn}\left(e_{2}(s)\right)\right) \mathrm{d} s
$$

with the adaptive update law

$$
\dot{\hat{\Theta}}(t)=\frac{1}{\gamma} \operatorname{sgn}\left(e_{2}^{T}(t)\right) \rho(t),
$$

where design parameters $k_{s}, \gamma \in \mathrm{R}$ are positive control gains, and $\hat{\Theta}(t) \in \mathrm{R}$ is the parameter estimation of $\Theta$ which will be specified later. The second term in (19) is used to ensure that $\mu(0)=0 . \mu(t)$ does not depend on the unmeasurable filtered tracking error term $\rho$, but its time derivative can be expressed as a function of $\rho$. Taking the time derivative of $\mu(t)$, one has

$$
\begin{aligned}
\dot{\mu}(t) & =\left(k_{s}+1\right) \dot{e}_{2}(t)+\left(k_{s}+1\right) \alpha_{2} e_{2}(t)+\hat{\Theta}(t) \operatorname{sgn}\left(e_{2}(t)\right) \\
& =\left(k_{s}+1\right) \rho(t)+\hat{\Theta}(t) \operatorname{sgn}\left(e_{2}(t)\right) .
\end{aligned}
$$

Substituting (21) into (18) results in

$$
M_{1} \dot{\rho}=-\left(k_{s}+1\right) \rho-\hat{\Theta}(t) \operatorname{sgn}\left(e_{2}\right)-\frac{1}{2} \dot{M}_{1} \rho-e_{2}+\Gamma,
$$

where $\Gamma(z, \dot{z}, t)=\dot{\Phi}_{1}+\Upsilon-\frac{1}{2} \dot{M}_{1} \rho+e_{2} \in \mathrm{R}^{n-m}$. Defining

$$
\Gamma_{d}=\frac{\partial \Phi_{1}}{\partial z_{d}} \dot{z}_{d}+\frac{\partial \Phi_{1}}{\partial \dot{z}_{d}} \ddot{z}_{d}+\frac{\partial \Phi_{1}}{\partial \ddot{z}_{d}} z_{d}^{(3)} .
$$

Noting that $\Phi_{1}$ is an $\mathcal{C}^{2}$ vector function and $z_{d}^{(i)} i=0, \cdots, 4$ are all bounded, there must exist two unknown positive constants $B_{1}$ and $B_{2}$, such that

$$
\left\|\Gamma_{d}\right\| \leq B_{1}, \quad\left\|\dot{\Gamma}_{d}\right\| \leq B_{2} .
$$

\section{MAIN RESUlTS}

Theorem 1 Consider the nonholonomic mechanical system described by (1) and (2), subjects to Assumptions 1 and 2. Given a desired trajectory $z_{d}(t)$ which satisfies the constraint equation (2), using the control laws (15), (19) and (20), the following hold: (i) All the states of the closed-loop system are globally bounded. (ii) Tracking error $e_{1}$ and $\dot{e}_{1}$ converge to zero as $t \rightarrow \infty$.

Proof. Let $D \in \mathrm{R}^{3(n-m)+2}$ be a domain containing $y(t)=0$, where $y(t) \in \mathrm{R}^{3(n-m)+2}$ is defined as $y(t)=$ $\left[x^{T}(t), \tilde{\Theta}(t), \sqrt{P(t)}\right]^{T}, x(t) \in \mathrm{R}^{3(n-m)}$ is defined as $x(t)=$ $\left[e_{1}^{T}, e_{2}^{T}, \rho^{T}\right]^{T}$, and $\tilde{\Theta}(t)=\Theta-\hat{\Theta}(t)$ represents the parameter estimation error. $P(t) \in \mathrm{R}$ is defined as

$$
P(t)=\Theta\left\|e_{2}(0)\right\|-e_{2}(0)^{T} \Gamma_{d}(0)-\int_{0}^{t} L(s) \mathrm{d} s,
$$

where the auxiliary function $L(t)$ is defined as

$$
L(t)=\rho^{T}\left(\Gamma_{d}(t)-\Theta \operatorname{sgn}\left(e_{2}\right)\right) .
$$

Selecting $\Theta=B_{1}+\frac{1}{\alpha_{2}} B_{2}+1$, by taking the same manipulations as Appendix $\mathrm{A}$ in [13], there is

$$
\int_{0}^{t} L(s) \mathrm{d} s \leq \Theta\left\|e_{2}(0)\right\|-e_{2}(0)^{T} \Gamma_{d}(0) .
$$

Hence, $P(t) \geq 0$.

Now, choose a candidate lyapunov function

$$
V(y, t)=e_{1}^{T} e_{1}+\frac{1}{2} e_{2}^{T} e_{2}+\frac{1}{2} \rho^{T} M_{1} \rho+P+\frac{\gamma}{2} \tilde{\Theta}^{2} .
$$

Taking the time derivative of $V$ along solutions of (10), noting the definition of $\dot{\hat{\Theta}}$ and substituting (11), (13) and (22) into it, we immediately get

$\dot{V} \leq-2 \alpha_{1}\left\|e_{1}\right\|^{2}-\alpha_{2}\left\|e_{2}\right\|^{2}-\left(k_{s}+1\right)\|\rho\|^{2}+2 e_{1}^{T} e_{2}+\rho^{T} \tilde{\Gamma}$.

Since $\Gamma(t)$ is continuously differentiable, by mean valve theorem, one can acquire the upper bound of $\tilde{\Gamma}$ as follows [13]:

$$
\|\tilde{\Gamma}\| \leq \varphi(\|x\|)\|x\|,
$$

where $\varphi: \mathrm{R}^{+} \rightarrow \mathrm{R}^{+}$is an appropriate $\mathcal{K}$ function. By using the fact that $2 e_{1}^{T} e_{2} \leq\left\|e_{1}\right\|^{2}+\left\|e_{2}\right\|^{2}, \dot{V}$ can be simplified as

$$
\dot{V} \leq-\lambda\|x\|^{2}-k_{s}\|\rho\|^{2}+\varphi(\|x\|)\|\rho\|\|x\|,
$$

where $\lambda=\min \left\{2 \alpha_{1}-1, \alpha_{2}-1,1\right\}$, and $\alpha_{1}, \alpha_{2}$ must be chosen to satisfy $\alpha_{1}>\frac{1}{2}, \alpha_{2}>1$.

Completing the squares for the third term in (24) gives

$$
\varphi(\|x\|)\|\rho\|\|x\| \leq k_{s}\|\rho\|^{2}+\frac{\varphi^{2}(\|x\|)\|x\|^{2}}{4 k_{s}},
$$

then the following expression can be obtained

$$
\dot{V} \leq-\lambda\|x\|^{2}+\frac{\varphi^{2}(\|x\|)\|x\|^{2}}{4 k_{s}} .
$$

Now, we define a compact set:

$$
N_{1}=\left\{y \in \mathrm{R}^{3(n-m)+2} \mid\|y\| \leq \varphi^{-1}\left(2 \sqrt{\lambda k_{s}}\right)\right\} .
$$

The inequality (25) shows $V(t) \leq V(0)$ in $N_{1}$, hence, all the the signals $e_{1}, e_{2}, \rho, \tilde{\Theta}$ on the right-hand side of function (23) are bounded in $N_{1}$. From the definition of $e_{1}, e_{2}, \rho, \tilde{\Theta}$, we know $\dot{e}_{1}=e_{2}-\alpha_{1} e_{1}, \dot{e}_{2}=\rho-\alpha_{2} e_{2}, \tilde{\Theta}=\Theta-\hat{\Theta}$, therefore, we can further get $\dot{e}_{1}, \dot{e}_{2}, \hat{\Theta} \in \mathcal{L}_{\infty}$ in $N_{1}$. The assumption that $z_{d}, \dot{z}_{d}, \ddot{z}_{d}$ are bounded can be used to conclude that $z, \dot{z}, \ddot{z}$ $\in \mathcal{L}_{\infty}$ in $N_{1}$. With $M_{1}, V_{1}, G_{1}$ are all known and bounded in $N_{1}$. Thereby $\tau_{1}, \mu \in \mathcal{L}_{\infty}$ in $N_{1}$ can be further obtained.

Then, let $N_{2} \subset N_{1}$ denotes a set defined as follows:

$$
N_{2}=\left\{y(t) \subset N_{1} \mid \delta_{2}(y)\|y\|^{2}<\delta_{1} \cdot\left(\varphi^{-1}\left(2 \sqrt{\lambda k_{s}}\right)\right)^{2}\right\} \text {, }
$$

where $\delta_{1}=\frac{1}{2} \min \{1, a\}, \delta_{2}(y)=\max \left\{1, \frac{1}{2} \bar{a}(y)\right\}$, and the definitions of $a$ and $\bar{a}(y)$ have been given in Assumption 1 . From expression (25), one obtains that there must exist an appropriate positive semidefinite function $U(y)=c\|x(t)\|^{2}$, such that $\dot{V} \leq-U(y)$. With Invariance-like Theorem (Theorem 8.4 of [14]) in mind, one can further get $U(y)=c\|x(t)\|^{2} \rightarrow$ 0 ast $\rightarrow \infty$. Based on the definitions of $x(t)$, one can finally gain $e_{1}(t), e_{2}(t), \rho(t) \rightarrow 0$ as $t \rightarrow \infty, \forall y(0) \in N_{2}$. From (13), we then know $\dot{e}_{1}(t), \dot{e}_{2}(t) \rightarrow 0$ as $t \rightarrow \infty, \forall y(0) \in N_{2}$.

On the other hand, from (17), it is evident that if $\rho(t)$, $e_{2}(t)$ and $\dot{e}_{1}(t)$ are all bounded, then $\mu(t)-\Phi_{1}$ is bounded. According to the boundedness of $S_{1}(q), S_{1}^{T}\left(\mu(t)-\Phi_{1}\right)$ is bounded. Substituting the control laws (15) and (19) into reduced order dynamic model (9) yields

$$
J(q)\left(\lambda-\lambda_{c}\right)=S_{1}^{T}\left(\Phi_{1}-\mu(t)\right)-M_{0} \ddot{e}_{1}-V_{0} \dot{e}_{1}=\omega(\cdot) .
$$

$\omega(\cdot)$ be a bounded function vector. Therefore, the force tracking error $\left(f-f_{d}\right)$ is bounded and can be adjusted by changing the feedback gain $k_{\lambda}$. Thus, the theorem is proved completely. 


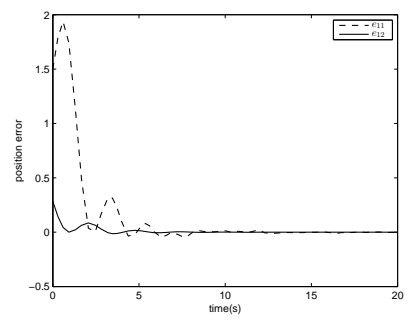

Fig. 1 The trajectories of $e_{1}(t)$

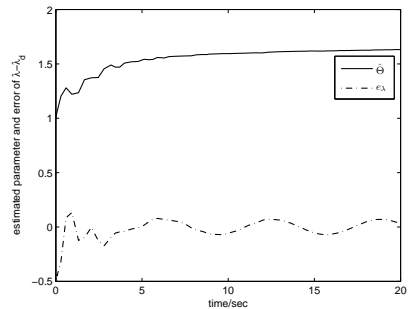

Fig. 3 The trajectories of $\hat{\Theta}(t), e_{\lambda}(t)$

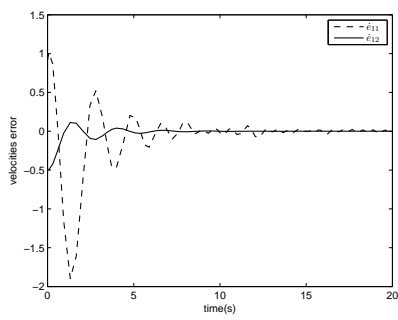

Fig. 2 The trajectories of $\dot{e}_{1}(t)$

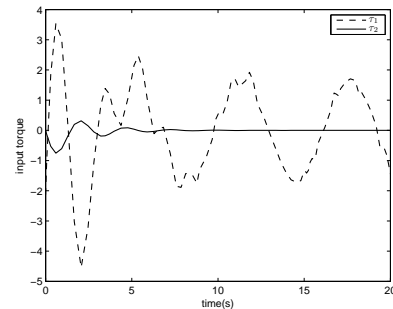

Fig. 4 The trajectories of $\tau(t)$

\section{Simulation}

Consider a boat with payload on a running river. The $x$-axis and $y$-axis denote the transverse direction and the downstream direction of the river, respectively. Here, we suppose the stream of the river only depends on transverse position $x$ in the simulation. The affine constraints can be obtained as follows:

$$
\cos \theta \dot{y}-\sin \theta \dot{x}=C(x) \cos \theta .
$$

We assume that the traveling direction velocity and the angular velocity of the boat can be controlled. The standard forms are given as follows:

$$
\begin{aligned}
M(q) & =\left[\begin{array}{ccc}
m+m_{0} & 0 & 0 \\
0 & m+m_{0} & 0 \\
0 & 0 & I+I_{0}
\end{array}\right], \\
V(q, \dot{q}) & =0, \quad G(q)=0, \\
J^{T}(q) & =\left[\begin{array}{lll}
\cos q_{3}, & -\sin q_{3}, & 0
\end{array}\right], A(q)=C\left(q_{2}\right) \cos q_{3},
\end{aligned}
$$

where $m$ is the mass of the boat and $I$ is the inertia of the boat, $m_{0}$ denotes the unknown mass of the payload and $I_{0}$ denotes the unknown inertia of the payload. For the sake of simplicity, select $m=1, I=1, C\left(q_{2}\right)=q_{2}$.

For the given $J(q), S(q)$ and $\eta(q)$, the desired trajectory $q_{d}=\left[\sin t-\cos t, \sin t, \frac{\pi}{4}\right]^{T}$ satisfies kinematic constraint $J^{T}\left(q_{d}\right) \dot{q}_{d}=A\left(q_{d}\right)$ and diffeomorphism transform $\dot{q}_{d}=$ $S\left(q_{d}\right) \dot{z}_{d}+\eta\left(q_{d}\right)$ with $z_{d}=\left[\sqrt{2} \sin t+2, \frac{\pi}{4}\right]^{T}$. The control objective is to determine an adaptive feedback control so that the trajectory $z$ follows $z_{d}$, and $\lambda$ is bounded.

In the simulation, suppose $m_{0}=0.1, I_{0}=0.1$, chose $\alpha_{1}=$ $1, \alpha_{2}=2, k_{s}=1, k_{\lambda}=2, \gamma=10$, and select $z_{1}(0)=$ $z_{2}(0)=0.5, \dot{z}_{1}(0)=\dot{z}_{2}(0)=0.5, \hat{\Theta}(0)=1$. The results of the simulation are shown in Figs $2 \sim 5$. Fig. 2 shows the position tracking errors of $z(t)-z_{d}(t)$ converge to zero, Fig. 3 shows the velocity tracking errors of $\dot{z}(t)-\dot{z}_{d}(t)$ converge to zero, Fig. 4 shows both state $\hat{\Theta}(t)$ and the tracking error of $e_{\lambda}$ are bounded. It can be seen that the control inputs shown in Fig. 5 are bounded.

\section{CONCLUSIONS}

In this paper, the motion and force tracking problem is addressed for a class of uncertain nonholonomic mechanical systems. The controller guarantees that the configuration state of the system semi-global asymptotically tracks to the desired trajectory and the force tracking error is bounded with a controllable bound.

\section{ACKNOWLEDGMENT}

This work was supported by National Natural Science Foundation of China under Grant 61273091 and 61304059, the Project of Taishan Scholar of Shandong Province, the Ph. D. Programs Foundation of Ministry of Education of China, the Fundamental Research Funds for the Central Universities under Grant CXLX12_0096.

\section{REFERENCES}

[1] Y.Q. Wu, B. Wang, G.D. Zong, "Finite-time tracking controller design for nonholonomic systems with extended chained form". IEEE Transactions on Circuits and Systems II: Express Briefs, vol. 50, pp. 798-802, November 2005

[2] M. Markus, "Robust tracking control of nonholonomic dynamic systems with application to the bi-steerable mobile robot". Automatica, vol. 44, pp. 2588-2592, October 2008.

[3] W.J. Dong, W.L. Xu, W. Huo, "Trajectory tracking control of dynamic nonholonomic systems with unknown dynamics". International Journal of Robust and Nonlinear Control, vol. 9, pp. 905-922, 1999.

[4] T. Fukao, H. Nakagawa, N. Adachi, "Adaptive tracking control of a nonholonomic mobile robot". IEEE Transactions on Robotics and Automation, vol. 16, pp. 609-615, May 2000.

[5] G.L. Ju, Y.Q. Wu, W.H. Sun, "Adaptive output feedback asymptotic stabilization of nonholonomic systems with uncertainties". Nonlinear Analysis: Theory, Methods and Applications, vol. 71, pp. 5106-5117, November 2009

[6] W.J. Dong, W.L. Xu, "Adaptive tracking control of uncertain nonholonomic dynamic system". IEEE Transactions on Automatic Control, vol. 46, pp. 450-454, May 2001.

[7] Z.J. Li, J Luo, "Adaptive robust dynamic balance and motion controls of mobile wheeled inverted pendulums". IEEE Transactions on Control Systems Technology, vol. 17, pp. 233-241, January 2009.

[8] M. Oya, C.Y. Su, R. Katoh, "Robust adaptive motion/force tracking control of uncertain nonholonomic mechanical systems". IEEE Transactions on Automatic Control, vol. 19, pp. 175-181, January 2003.

[9] C.Y. Su, Y. Stepanenko, "Robust motion/force control of mechanical systems with classical nonholonomic constraints". IEEE Transactions on Automatic Control, vol. 39, pp. 609-614, May 1994.

[10] T. Kai, "Modeling and passivity analysis of nonholonomic Hamiltonian systems with rheonomous affine constraints". Communications in Nonlinear Science and Numerical Simulation, vol. 17, pp. 3446-3460, August 2012.

[11] T. Kai, "Mathematical modelling and theoretical analysis of nonholonomic kinematic systems with a class of rheonomous affine constraints". Applied Mathematical Modelling, vol. 36, pp. 3189-3200, July 2012.

[12] S.S. Ge, T.H. Lee, C.J. Harris. Adaptive neural network control of robust manipulators. Singapore: Word Scientific, 1998.

[13] B. Xian, D.M. Dawson, M.S. Queiroz, "A continuous asymptotic tracking control strategy for uncertain multi-input nonlinear systems". IEEE Transactions on Automatic Control, vol. 49, pp. 1206-1211, July 2004.

[14] H.K. Khalil, Nonlinear systems (3rd edition). New Jersey: Prentice Hall, 2002. 